GNPHE/0902

\title{
On Local F-theory Geometries and Intersecting D7-branes
}

\author{
Rachid Ahl Laamara ${ }^{1,4 *}$, Adil Belhaj ${ }^{2,4 \dagger}$, Luis J. Boya ${ }^{3 \ddagger}$ Antonio Segui ${ }^{3 \S}$ \\ ${ }^{1}$ Lab/UFR-Physique des Hautes Energies, Faculté des Sciences, Rabat, Morocco \\ ${ }^{2}$ Centre National de l'Energie, des Sciences et des Techniques Nucléaires, CNESTEN \\ Cellule Science de la Matière, Rabat, Morocco \\ ${ }^{3}$ Departamento de Física Teórica, Universidad de Zaragoza, E-50009-Zaragoza, Spain \\ ${ }^{4}$ Groupement National de Physique des Hautes Energies, GNPHE \\ Siège focal: Faculté des Sciences, Rabat, Morocco
}

January 8, 2019

\begin{abstract}
We discuss local F-theory geometries and theirs gauge theory dualities in terms of intersecting D7-branes wrapped four-cycles in Type IIB superstring. The manifolds are built as elliptic K3 surface fibrations over intersecting $F_{0}=\mathbf{C P}^{\mathbf{1}} \times \mathbf{C P}^{\mathbf{1}}$ base geometry according to $A D E$ Dynkin Diagrams. The base is obtained by blowing up the extended $A D E$ hyper-Kähler singularities of eight dimensional manifolds considered as sigma model target spaces with eight supercharges. The resulting gauge theory of such local F-theory models are given in terms of Type IIB D7-branes wrapped intersecting $F_{0}$. The four dimensional $N=1$ anomaly cancelation requirement translates into a condition on the associated affine Lie algebras.
\end{abstract}

KEYWORDS: F-theory, Type II superstrings, $A D E$ singularities, Supersymmetric Sigma Models.

*doctorants.lphe@fsr.ac.ma

†belhaj@unizar.es

¥luisjo@unizar.es

${ }^{\S}$ segui@unizar.es 


\section{Introduction}

A very nice way to get supersymmetric gauge theory from superstrings, $\mathrm{M}$ or F-theory, is to use the so-called geometric engineering method which is based on singular manifold compactifications [1]-[7]. In this way, the internal manifold is a K3 fibration over a base space $B$ which depends on the theory in question. The gauge group $G$ comes from the singularities of the fiber while the matter is obtained from non-trivial geometries in the base [8]. In the case of Type II superstrings with eight supercharges, the complete set of physical parameters of the corresponding quantum field theory (QFT) is related to the geometric moduli space of the internal manifold. The latter is realized as a singular K3 fibration over a $\mathbf{C P}^{\mathbf{1}}$ complex curve or a collection of intersecting $\mathbf{C P}^{\mathbf{1}}$ curves according to Dynkin geometries. The corresponding four-dimensional (4D) $N=2$ QFT are represented by quiver graphs similar to Dynkin diagrams of ordinary, affine, and indefinite Lie algebras [2, 4, 9, 10, 11].

More recently four dimensional gauge theories with only four supercharges, obtained from local F-theory models, have attracted a lot of attention, since they have connections with the physics of standard model, grand unification theory (GUT), and Large Hadron Collider (LHC) [12]-[22]. For instance, in this study of F-theory GUT models involves geometric singularities which are related to D7-branes of Type IIB superstring and are localized in two dimensional transverse space. It has been shown that the gauge fields of the MSSM are obtained from the eight-dimensional worldvolume of a seven-brane wrapping del Pezzo surfaces with a GUT gauge group. The latter can be broken to $S U(3)_{C} \times S U(2)_{L} \times U(1)_{Y}$ via an internal flux through the seven-brane in the $U(1)_{Y}$ direction of the GUT. Alternative study has been also done for M-theory on seven dimensional manifolds admitting $G_{2}$ metrics [23, 24, 25].

The aim of this work is to contribute to F-theory compactification activities. In particular, we engineer geometrically quiver gauge theories with bi-fundamental matters from local Ftheory models. First, we construct such local models as K3 fibrations over a four-dimensional base space. The base geometry is obtained by blowing up the extended $A D E$ hyper-Kähler singularities of eight dimensional manifolds considered as sigma model target spaces with eight supercharges. Actually, this extends the blow up of the ordinary $A D E$ Kähler singularities of local K3 surfaces described by sigma models with only four supercharges. Up some details, the base in local F-theory geometry has been identified with intersecting $F_{0}=\mathbf{C P}^{\mathbf{1}} \times \mathbf{C P}^{\mathbf{1}}$ according to the $A D E$ Dynkin diagrams. This means that instead of having intersecting $\mathbf{C P}^{\mathbf{1}}$ curves as in the case of $N=2$ geometric engineering, we have now intersecting $F_{0}$ in the base of local F-theory Calabi-Yau fourfolds. The corresponding gauge theories have been discussed 
in terms of intersecting D7-branes in Type IIB superstring. This allows us to give a product of $S U$ gauge groups with bi-fundamental matter encoded in affine $A D E$ Dynkin geometries required by $4 \mathrm{D}$ anomaly cancelation condition. In this way, the rank of each $S U$ gauge group is related to the Coxter number on the corresponding node. In the end of this work, a discussion on the standard model gauge group is given in terms of twisted non simply laced $\hat{G}_{2}^{2}$ base geometry.

The organization of this paper is as follows. In section 2, we engineer geometrically local F-theory models with $A D E$ base geometries. Using $N=4$ sigma model, the manifolds are constructed as elliptic K3 surfaces fibred over intersecting $F_{0}=\mathbf{C P}^{\mathbf{1}} \times \mathbf{C P}^{\mathbf{1}}$ according to the $A D E$ Dynkin diagrams. In section 3, we discuss the corresponding gauge group and matter content in terms of intersecting D7-branes in Type IIB superstring. In particular we show how the anomaly cancelation condition translates into a condition on the associated affine $A D E$ geometries in the base of our local F-theory geometry. A speculation on the standard model gauge symmetry is given in section 4 . In particular, the gauge group $G=U(1) \times S U(2) \times S U(3)$

is discussed in terms of the twisted non simply laced $\hat{G}_{2}^{2}$ quiver diagram.

\section{On Constructing Local F-theory Models}

In this section, we engineer local F-theory models leading to bi-fundamental matter in four dimensions with $N=1$ supersymmetry. This will be given by an elliptic K3 fibration over a four-dimensional base space $B_{4}$ with the following Hodge number condition

$$
h^{2,0}\left(B_{4}\right)=0
$$

As in Type IIA superstring and M-theory case mentioned in the introduction, the incorporation of the matter may be achieved by introducing a non-trivial geometry in the base space $B_{4}$. This leads us to consider a local model with intersecting base geometry in order to produce a product gauge group with bi-fundamental matter.

Our strategy in this section consists of two steps. First we recall some aspects of F-theory. Then we turn to the building of local F-theory geometries. Particular emphasis will be put on the four-dimensional $A D E$ base geometry. The later is embedded in a real eight dimensional manifolds admitting hyper-Kähler metrics. The $A D E$ part will correspond to a 4-dimensional geometry obtained by resolving $A D E$ extended hyper-Kähler singularities studied in [26]. 


\subsection{Generalities on F-theory}

F-theory defines a non-perturbative vacuum of Type IIB superstring theory in which the dilaton and axion fields of the superstring theory are considered dynamical [27, 28]. This introduces an extra complex modulus which is interpreted as the complex parameter of an elliptic curve thereby introducing a non-perturbative vacuum of the Type IIB superstring in a twelve-dimensional space-time. Following Vafa [27, one may interpret the complex scalar field $\tau$ of Type IIB superstring as the complex structure of an extra torus $T^{2}$ resulting in the aforementioned twelve-dimensional model. From this point of view, Type IIB superstring theory may be seen as the compactification of F-theory on $T^{2}$. Starting from F-theory, one can similarly look for new superstring models in lower dimensions obtained by compactifications on elliptically fibered Calabi-Yau manifolds. For example, the eight-dimensional F-theory on elliptically fibred K3 is obtained by taking a two-dimensional complex compact manifold given by

$$
y^{2}=x^{3}+f(z) x+g(z)
$$

where $f$ and $g$ are polynomials of degree 8 and 12, respectively. One varies the $\tau$ over the points of a compact space which is taken to be a Riemann sphere $\mathbf{C P}^{\mathbf{1}}$ parameterized by the local coordinate $z$. In other words, the two-torus complex structure $\tau(z)$ is now a function of $z$ as it varies over the $\mathbf{C P}^{\mathbf{1}}$ base of the above K3 surface. The above compact manifold generically has 24 singular points corresponding to $\tau(z) \rightarrow \infty$. These singularities have a remarkable physical interpretation as each one of the 24 points is associated with the location of a D7-brane in non-perturbative Type IIB superstring theory.

In the following, we will consider F-theory on elliptic K3 fibration over $B_{4}$. In particular, we will focus our attention on the base $B_{4}$ which gives information about the gauge group form in $4 \mathrm{D}$. This gauge theory shares similar features as the quiver gauge models which describe D-branes at singularities in Type II superstrings on local Calabi-Yau threefolds.

\subsection{Base Geometry from $A D E$ Extended Hyper-Kähler Singulari- ties}

Our choice of the base $B_{4}$ is motivated by the construction of intersecting base geometries involved in the geometric engineering of Type IIA local models in $4 D$ with $N=2$ [1, 2, 8]. This has been obtained by blowing up the ordinary $A D E$ singularties of the K3 surface which have a nice physical representation in terms of sigma model with four supercharges [29, 30, 31]. 
However, the idea of the present construction is to consider intersecting geometries in the base $B_{4}$ by resolving of the so-called extended $A D E$ hyper-Kähler singularities of eight dimensional manifolds described by $N=4$ sigma models [26]. To do so, let us first recall the ordinary $A D E$ singularities in the case of $N=2$ sigma model. Indeed, consider the leading example: $A_{1}$ singularity. This has a nice physical representation in terms of two-dimensional $N=2$ linear sigma model with only $U(1)$ gauge group and three chiral fields $\phi_{i}, i=1,2,3$ with charges $q_{i}=(1,-2,1)$ satisfying the local Calabi-Yau condition

$$
\sum_{i} q_{i}=1-2+1=0 .
$$

The $U(1)$ gauge invaraint $x=\phi_{1}^{2} \phi_{2}, y=\phi_{3}^{2} \phi_{2}$ and $z=\phi_{1} \phi_{2} \phi_{3}$ satisfies the usual the $A_{1}$ singularity

$$
x y=z^{2} .
$$

The corresponding $D$-term bosonic potential $U\left(\phi_{1}, \phi_{2}, \phi_{3}\right)$, in supersymmetric theories with four supercharges, reads as

$$
U\left(\phi_{1}, \phi_{2}, \phi_{3}\right)=\left(\left|\phi_{1}\right|^{2}+\left|\phi_{3}\right|^{2}-2\left|\phi_{2}\right|^{2}-R\right)^{2} .
$$

In this equation, $R$ is the coupling parameter of the $U(1)$ Fayet-Iliopoulos (FI) term one may introduce in the Lagrangian model. In the superfield language, the action then reads

$$
S(\Phi, V)=\int d^{2} x d^{4} \theta \bar{\Phi} e^{V} \Phi-R \int d^{2} x d^{4} \theta V,
$$

where $\Phi$ and $V$ are respectively the chiral and gauge superfields. The presence of the FI term resolves the singularity of the potential $U\left(\phi_{1}, \phi_{2}, \phi_{3}\right)$. Geometrically, this corresponds to replacing the singular point $x=y=z=0$ by a $\mathbf{C P}^{1}$ described by

$$
\left|\phi_{1}\right|^{2}+\left|\phi_{3}\right|^{2}=R \text {. }
$$

Note that $\phi_{2}$ defines the cotangent direction over $\mathbf{C P}^{\mathbf{1}}$ of the deformed $A_{1}$ ALE space1

The above $N=2$ sigma model can be extended to $N=4$ supersymmetric gauge theory with eight supercharges describing eight dimensional manifolds with $A D E$ hyper-Kähler singularities. This involves $U(1)^{r}$ gauge symmetry and $r+2$ hypermultiplets with a matrix charge $Q_{i}^{a}$, which can be identified with the Cartan matrices of $A D E$ Lie algebras [26]. The $N=4$ D-flatness equations of such models are generally given by the moduli space of the hypermultiplets vacua

$$
\sum_{i=1}^{r+2} Q_{i}^{r}\left[\phi_{i}^{\alpha} \bar{\phi}_{i \beta}+\phi_{i}^{\beta} \bar{\phi}_{i \alpha}\right]=\vec{\xi}_{a} \vec{\sigma}_{\beta}^{\alpha},
$$

\footnotetext{
${ }^{1}$ More general study for $A D E$ singularities of K3 surface can be found in the appendix.
} 
where $\phi_{i}^{\alpha}$ 's denote $r+2$ component field doublets of hypermultiplets, $\vec{\xi}_{a}$ are $r$ FI 3-vector couplings rotated by $S U(2)$ symmetry, and $\vec{\sigma}_{\beta}^{\alpha}$ are the traceless $2 \times 2$ Pauli matrices. Equations (8) deal with the hypermultiplet branch and give a gauge invariant hyper-Kähler target space. For each $U(1)$ factor of the $U(1)^{r}$ gauge group, they involve a triplet of FI parameters. Note also that (8) have a manifest $S U(2)_{R}$ symmetry which is absent in $N=2$ sigma model discussed before.

Using the $S U(2)_{R}$ transformations

$$
\phi^{\alpha}=\varepsilon^{\alpha \beta} \phi_{\beta}, \quad \overline{\left(\phi^{\alpha}\right)}=\bar{\phi}_{\alpha}, \quad \varepsilon_{12}=\varepsilon^{21}=1
$$

and replacing the Pauli matrices by their expressions, the identities (8) can be split as follows

$$
\begin{aligned}
\sum_{i=1}^{r+2} Q_{i}^{a}\left(\left|\phi_{i}^{1}\right|^{2}-\left|\phi_{i}^{2}\right|^{2}\right) & =\xi_{a}^{3} \\
\sum_{i=1}^{r+2} Q_{i}^{a} \phi_{i}^{1} \bar{\phi}_{i}^{2} & =\xi_{a}^{1}+i \xi_{a}^{2} \\
\sum_{i=1}^{r+2} Q_{i}^{a} \phi_{i}^{2} \bar{\phi}_{i}^{1} & =\xi_{a}^{1}-i \xi_{a}^{2} .
\end{aligned}
$$

We will see later that the solution of these equations defines cotangent bundles over intersecting 4-cycles. Actually, this extends the result of the ordinary $A D E$ singularities of the K3 surface where it appears intersecting 2-cycles. These 4-cycles will be identified with the base of local F-theory models allowing us to produce a product of $S U$ gauge groups with bi-fundamental matter. The latter can be encoded in quiver diagrams similar to Dynkin graphs. For simplicity reasons we center on the $A_{r}$ geometry. In this case, we have a matrix charge of the form

$$
Q_{i}^{a}=\delta_{i-1}^{a}-2 \delta_{i}^{a}+\delta_{i+1}^{a}
$$

To handle the corresponding D-terms equations, it should be interesting to note that they are quite similar to the analysis of $N=2$ sigma model discussed in the appendix. Indeed, forget for the while the two last equations of (10) for $A_{r}$ model. Setting $\xi_{a}^{3}=R_{a}-P_{a}$ and taking the condition that $R_{a}>P_{a}$, one may put the first equation of (10) for $A_{r}$ model into the two following equations

$$
\begin{aligned}
& \left|\phi_{a-1}^{1}\right|^{2}+\left|\phi_{a+1}^{1}\right|^{2}-2\left|\phi_{a}^{1}\right|^{2}=R_{a} \\
& \left|\phi_{a-1}^{2}\right|^{2}+\left|\phi_{a+1}^{2}\right|^{2}-2\left|\phi_{a}^{2}\right|^{2}=P_{a}
\end{aligned}
$$

Let us comment these equations: 
- They describe two orthogonal copies of $A_{r}$ models. In the case where $R_{a}=0$ and $P_{a}$ positive definite, or $R_{a}$ positive definite and $P_{a}=0$, one of the $A_{r}$ models becomes singular. If $R_{a}=P_{a}=0$ both of them are singular.

- For $R_{a}$ positive definite and $P_{a}$ positive definite, we have the blown up of the two $A_{r}$ singularities where each $A_{r}$ model has four charges obtained by breaking $N=4$ supersymmetry to $N=2$.

To better understand the geometry given by (10) for $A_{r}$ model, let us deal with the leading example corresponding to $r=1$. This involves one $U(1)$ gauge group with three hypermultiplets $\phi_{i}$ of charges $Q_{i}=(1,-2,1)$. In this case, (12) reduce to

$$
\begin{aligned}
& \left|\phi_{1}^{1}\right|^{2}+\left|\phi_{3}^{1}\right|^{2}-2\left|\phi_{2}^{1}\right|^{2}=R \\
& \left|\phi_{1}^{2}\right|^{2}+\left|\phi_{3}^{2}\right|^{2}-2\left|\phi_{2}^{2}\right|^{2}=P .
\end{aligned}
$$

These equations describe the cotangent bundle over the Hirzebruch surface $F_{0}=\mathbf{C P}^{\mathbf{1}} \times \mathbf{C P}^{\mathbf{1}}$. Indeed, putting $\phi_{2}^{1}=\phi_{2}^{2}=0$, (13) become

$$
\begin{aligned}
& \left|\phi_{1}^{1}\right|^{2}+\left|\phi_{3}^{1}\right|^{2}=R \\
& \left|\phi_{1}^{2}\right|^{2}+\left|\phi_{3}^{2}\right|^{2}=P .
\end{aligned}
$$

These two equations define the Hirzebruch surface $F_{0}=\mathbf{C P}^{1} \times \mathbf{C P}^{1}$ generalizing (77). Now we come back to the two last equations of (10) and see theirs roles. For simplicity reason, let us consider the case of $U(1)$ gauge symmetry and assume that the corresponding charges $Q_{i}$ are all positive which can be obtained by possible exchanging some chiral fields in the generic case. Putting $\xi_{i}^{2}=\xi_{i}^{3}=0$ and introducing $x_{i}=\sqrt{Q_{i}} \phi_{i}^{1}$ and $y_{i}=\sqrt{Q_{i}} \phi_{i}^{2}=0$, the two last equations (10) can be rewritten as

$$
\begin{aligned}
& x_{i} \bar{y}_{i}=0 \\
& \bar{x}_{i} y_{i}=0 .
\end{aligned}
$$

They can be viewed as a scalar product showing two orthogonal variables. Based on this remark and the fact that $\phi_{2}^{1}$ and $\phi_{2}^{2}$ describe two orthogonal non compact directions over $F_{0}$, our total geometry is given by the cotangent fiber over $F_{0}$ base. Locally, up to an orbifold action the fiber can be identified with local K3 surfaces making contact with D7-brane physics in Type IIB superstring. By assumption, the cotangent fiber can be divided by subgroups of $S U(2)$. If we denote this subgroup by $\Gamma$, the fiber looks like

$$
\mathbf{C}^{2} / \Gamma
$$


leading to $A D E$ gauge symmetries in eight dimensions [27].

The story is similar for the general $A_{r}$ geometry where we obtain intersecting $F_{0}$ 's where all $\xi^{a}$ s are no zero. We thus expect to obtain the cotangent bundle over $r$ intersecting $F_{0}=$

$\mathbf{C P}^{\mathbf{1}} \times \mathbf{C P}^{\mathbf{1}}$ according to $A_{r}$ Dynkin diagrams. This means that the base geometry, of the cotangent bundle, consists of $r$ intersecting $F_{0}=\mathbf{C P}^{\mathbf{1}} \times \mathbf{C P}^{\mathbf{1}}$ arranged as shown here

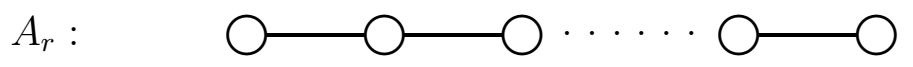

where the nodes represent $F_{0}$, while their intersections are represented by the links. This generalizes the case of $N=2$ sigma model where each node is associated with a $\mathbf{C P}^{\mathbf{1}}$ of the $A_{r}$ deformed singularity. We will conclude this construction by noting that this analysis for $A_{r}$ model may be extended to the others $D E$ Lie algebras where the corresponding geometries are classified by Dynkin graphs.

Having constructed the base geometry of our local F-theory model as intersecting $F_{0}=\mathbf{C P}^{\mathbf{1}} \times$ $\mathbf{C P}^{\mathbf{1}}$ according to $A D E$ Dynking graphs, we will discuss the corresponding quiver gauge theory in four dimensions.

\section{Intersecting D7-branes and Bi-fundamental Matter}

Our analysis here will be based on a dual Type IIB superstring description in terms of D7-brane backgrounds. In addition to D7-branes, a F-theory background may also contain D3-branes localized at some singular points. However, for reasons of simplicity, only the world volume of D7-branes will play a role in models we deal with. Theses branes wrap 4-cycles and fill the four-dimensional Minkowski space. Indeed, a local description of F-theory near the $A_{n-1}$ singularity of the K3 surfaces is equivalent to $n$ units of D7-branes in Type IIB dual version in eight dimensions [27]. This can be obtained by taking $\Gamma$ as

$$
\Gamma=Z_{n}
$$

On each D7-brane we have a $U(1)$ symmetry. When the $n$ D7-branes approach each other, the gauge symmetry is enhanced from $U(1)^{\otimes n}$ to $S U(n)$ [27]. This can be extended to arbitrary gauge group involving exceptional D7-branes [18]. An extra compactification of F-theory down to four-dimensional space-time is equivalent, in Type IIB superstring side, to wrapping D7-branes on 4-cycles. Here they will can be identified with intersecting $\mathbf{C P}^{\mathbf{1}} \times \mathbf{C P}^{\mathbf{1}}$. Consider now $m$ different stacks of D7-branes. This brane configuration can be reinterpreted as 
singularities of type $A_{n_{i}-1}$ which can meet at a point where the singularity jumps to higher gauge group. Note that each stack contains $n_{i}$ units of D7-branes. Identifying $m$ with the number of intersecting $\mathbf{C P}^{\mathbf{1}} \times \mathbf{C P}^{\mathbf{1}}$ and using the result of the geometric engineering in Type II superstrings and M-theory on $G_{2}$ manifolds and assuming that each stack of D-branes wrap one $F_{0}$, the the gauge group corresponding to such configurations can be described by

$$
G=\bigotimes_{i=1}^{m} S U\left(n_{i}\right)
$$

The $n_{i}$ which are integers can be specified by physical requirements. It turns out that in the case of $N=1$ models with four supercharges, these integers could be fixed by the anomaly cancelation condition [32, 33, 34]. They should form a null vector of a matter matrix $I_{i j}$ :

$$
\sum_{i=1}^{m} I_{i j} n_{i}=0 .
$$

As in the $N=2$ scenario [1, 2, 10, 11], the gauge group and matter depend on the intersection matrix $I_{i j}$. Recall that the intersection matrix of $n$ real dimensional sub-lagrangian manifolds considered as the blowup of singularities in $n$-dimensional Calabi-Yau manifolds is symmetric for $n$ even and antisymmetric for $n$ odd. In more general geometries, the intersection matrix may be written as a linear combination of a symmetric and antisymmetric term. From the obvious similarity with the $N=2$ scenario it is not surprising to to see some analogs with $A D E$ diagrams in eight-dimensional hyper-Kähler manifolds that we considered in the previous section. Indeed, the intersection theory assigns the intersection number to complex surfaces inside of such a manifold. For example, the self-intersection of the zero section in the cotangent bundle of $F_{0}$ is equal to its minus Euler characteristic, i.e. -4. Consider now a lattice of compact 4-cycles generated by $F_{0}^{i}$. Assume that $F_{0}^{i}$ intersects $F_{0}^{i+1}$ at two points. This can be supported by the fact that each $\mathbf{C P}^{\mathbf{1}}$ inside $F_{0}^{i}$ intersects just one $\mathbf{C P}^{\mathbf{1}}$ in the next $F_{0}^{i+1}$. In this way, the intersection numbers of the $F_{0}$ 's can be given by

$$
\begin{gathered}
F_{0}^{i} \cdot F_{0}^{i}=-4 \\
F_{0}^{i} \cdot F_{0}^{i+1}=2,
\end{gathered}
$$

with others vanishing. This means that $F_{0}^{i}$ does not intersect $F_{0}^{j}$ if $|j-i|>1$. Endowed with this intersection form, the lattice of compact 4-cycles can be identified with the root lattice of the $A D E$ Lie groups, up to a multiplication factor. A nice geometric interpretation for $A D E$ diagrams could be also obtained by looking at symplectic resolutions of finite quotients $V / G$ where $G$ is a finite subgroup of $S p(2)$ generated by symplectic reflections (i.e. elements $g$ with fixed locus of complex codimension 2). It would be worth investigating such geometries. 
From this discussion, we see that the information on our intersection geometry is naturally encoded in the Cartan matrix $K_{i j}$ of Lie Algebras. The latters are classified into three categories [35]:

1. Finite type $(\operatorname{det} K>0)$. In this case, there exists a real positive definite vector $u\left(u_{i}>\right.$ $0 ; i=1,2, \ldots)$ such that $K_{i j} u_{j}=v_{j}>0$.

2. Affine type, $\operatorname{corank}(K)=1$, det $K=0$. There exists a unique, up to a multiplicative factor, positive integer definite vector $n\left(n_{i}>0, i=1,2, \ldots\right)$ such that $K_{i j} n_{j}=0$.

3. Indefinite type $(\operatorname{det} K \leq 0), \operatorname{corank}(K) \neq 1$. There exists a real positive definite vector $u\left(u_{i}>0 ; i=1,2, \ldots\right)$ such that $K_{i j} u_{j}=-v_{i}<0$.

From this classification, it follows that the anomaly cancelation condition is translated into a condition on the affine Lie algebra. Indeed, (20) can be solved by

$$
\begin{aligned}
I_{i j} & \equiv-2 K_{i j} \\
n_{i} & \equiv s_{i} n \\
m & \equiv r+1
\end{aligned}
$$

where $K_{i j}$ are now the Cartan matrices of $A D E$ affine Lie algebras and $n$ is an arbitrary number. While $s_{i}$ are the Coxeter labels of the associated affine Lie algebra of rank $r$. As we have seen, the latter is related to the number of intersecting $F_{0}=\mathbf{C P}^{\mathbf{1}} \times \mathbf{C P}^{\mathbf{1}}$ that we should have in the base of our local F-theory geometry. The gauge group finally reads as

$$
G=\bigotimes_{i=1}^{r+1} S U\left(s_{i} n\right),
$$

with bi-fundamental chiral matter transforming in $\left(s_{i} n, s_{j} n\right)$ representations.

In the end of this section, we would like to note that it is possible to consider the two other Lie algebras by adding non trivial matters in order to satisfy the anomaly cancelation condition. We expect to have a similar analysis made for conformal invariance in the Type IIA geometric engineering method of $N=2$ in four dimensions studied in [10, 11]. It should be interesting to develop this issue in the future [36].

\section{Discussions}

In this work, we have engineered local F-theory geometries and four dimensional $N=1$ quiver gauge theories with bi-fundamental matters. The manifolds have been built as an elliptic K3 fibration over intersecting $F_{0}=\mathbf{C P}^{\mathbf{1}} \times \mathbf{C P}^{\mathbf{1}}$ surfaces according $A D E$ geometries. 
This intersecting base geometry can be obtained from the deformation of the extended of $A D E$ hyper-Kähler singularities of eight dimensional manifolds considered as target spaces of $N=4$ sigma model. Our main results may be summarized as follows:

(1) Using two-dimensional $N=4$ field theory with $U(1)^{r}$ gauge and $r+2$ hypermultiplets with a matrix charge, which can be identified, up some details, with $A D E$ Cartan matrices, we have constructed the $B_{4}$ geometry of local Calabi-Yau fourfolds. In particular, it has been shown that the corresponding target spaces are described by the cotangent bundle over intersecting $F_{0}=\mathbf{C P}^{1} \times \mathbf{C P}^{1}$ according to $A D E$ Dynkin diagrams. The latter has been identified with the base of our local F-theory geometries.

(2) By the help of intersecting D7-branes in Type IIB superstring, we have discussed the physics content of F-theory on such local Calabi-Yau fourfolds. In particular, we have engineered the gauge group and matter content. The $N=14 D$ anomaly cancelation condition has been converted into a condition on the associated affine Dynkin geometries in the base $B_{4}$.

Using this analysis the gauge group of standard model of electromagnetic, weak, and strong interactions could be discussed in terms of the folding of the Dynkin diagram of affine $E_{6}$ Lie algebra given by the following diagram

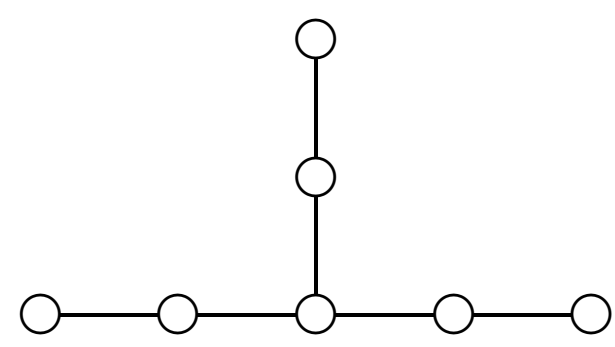

As well known, $E_{6}$ Lie algebra 2 has some relations with grand unified theory, since it has been considered as a possible gauge group which, after its breaking, gives rise to the gauge symmetry of the standard model [37]. However, our idea is bit different since instead of taking this symmetry as a singularity in the K3 surface fiber of F-thory compactification and break it to the usual standard model gauge group, we will consider it in the base geometry $B_{4}$. In this way, (23) becomes

$$
G=S U(3 n) \times S U(2 n)^{3} \times S U(n)^{3} .
$$

To get the gauge symmetry of the standard model, we proceed as follows:

- use the folding techniques of simply laced Dynkin diagram $(S L D D)$

\footnotetext{
${ }^{2}$ We thank J. L.Cortes for pointing us the reference [37.
} 
- take the limit $n=1$.

To do so, recall that the folding method gives rise non simply laced Dynkin diagrams (NSLDD). In particular, the corresponding diagram are obtained from the simply laced ones by identifying the Dynkin nodes which are permuted by a outer-automorphism group $\Gamma$. Formally, we can write this correspondence as follows

$$
S L D D / \Gamma \equiv N S L D D
$$

It turns out that, the Dynkin diagrams of affine $E_{6}$ has different realizations of the outerautomorphism group $\Gamma$ leading to different non simply laced diagrams. In the case of $\Gamma=Z_{3}$, we get the twisted $G_{2}^{2}$ affine Dynkin diagram. The latter has the following Coxter numbers

$$
s_{i}=(1,2,3)
$$

and can represented by the following Dynkin diagram

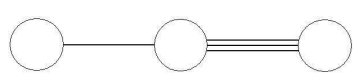

In this case, the (23) reduces to $G=S U(3) \times S U(2) \times U(1)$. It should be interesting to come back to this observation in the future.

Acknowledgments. RAL would like to thank A. Arhrib and S. Khalil for discussions on related subjects, scientific helps and kind hospitality at the BUE Centre for Theoretical Physics. AB would like to thank M. Asorey, B. Belhorma, J. L. Cortes, I. Dolgachev, L. B. Drissi, J. McKay, J. Rasmussen, E. H. Saidi and A. Sebbar for collaborations, discussions on related subjects and scientific helps. LJB and AS have been supported by CICYT (grant FPA-2006-02315) and DGIID-DGA (grant 2007-E24/2). This work has been supported by Fisica de altas energias: Particulas, Cuerdas y Cosmologia, A/9335/07.

\section{Apendix}

The local K3 surface with $A D E$ singularities can identified with the asymptotically locally Euclidean (ALE) space which is algebraically given by

$$
f_{A D E}(x, y, z)=0
$$


where $(x, y, z)$ are complex variables. The $A D E$ singularities are classified by

$$
\begin{array}{r}
A_{n-1}: f(x, y, z)=x y-z^{n} \\
D_{n}: f(x, y, z)=x^{2}+y^{2} z+z^{n-1} \\
E_{6}: f(x, y, z)=x^{2}+y^{3}+z^{4} \\
E_{7}: f(x, y, z)=x^{2}+y^{3}+y z^{3} \\
E_{8}: f(x, y, z)=x^{2}+y^{3}+z^{5} .
\end{array}
$$

They are all of them singular at $x=y=z=0$ since it is the only solution of $f_{A D E}=d f_{A D E}=$ 0 . These geometries can be 'desingularized' by deforming the complex structure of the surface or varying its Kähler structure. This consists in blowing up the singularity by a collection of intersecting complex curves. This means that we replace the singular point $(x, y, z)=(0,0,0)$ by a set of intersecting complex curves $\mathbf{C P}^{\mathbf{1}}$ (two-cycles). The nature of the set of intersecting $\mathbf{C P}^{\mathbf{1}}$ curves depends on the type of the singular surface one is considering. The smoothed $A D E$ surfaces share several features with the $A D E$ Dynkin diagrams. In particular, the intersection matrix of the complex curves used in the resolution of the $A D E$ singularities is, up to some details, minus the $A D E$ Cartan matrix $K_{i j}$. As in the case of $A_{1}$, the deformed $A D E$ Kähler geometries can also have nice physical interpretation in terms of $N=2$ sigma models. In general, these are described by a $U(1)^{r}$ gauge group with $(r+2)$ chiral multiples $\phi_{i}$ with $q_{i}^{a}$ vector charges satisfying the Calabi-Yau condition

$$
\sum_{i} q_{i}^{a}=0
$$

under which the gauge model flow in the infrared to $2 D N=2$ superconformal field theory. The $A D E$ spaces of classical vacua, in the absence of the sigma model superpotential, are given by

$$
U\left(\phi_{i}\right)=\sum_{i}\left(q_{i}^{a}\left|\phi_{i}\right|^{2}-R^{a}\right)^{2} .
$$

where $r$ is the rank of $A D E$ algebras in question. $q_{i}^{a}$, up to details, are proportional to the corresponding Cartan matrices $K_{a i}$. In the case of $A_{r}$ ALE space, we have $U(1)^{r}$ theory with $r+2$ chiral fields, with charges given by

$$
\begin{aligned}
q^{1} & =(1,-2,1,0,0,0, \ldots, 0), \\
q^{2} & =(0,1,-2,1,0,0, \ldots, 0), \\
q^{3} & =(0,0,1,-2,1,0, \ldots, 0), \\
& \cdots \\
q^{r} & =(0,0,0,0, \ldots, 1,-2,1) .
\end{aligned}
$$


In this case, the generators of gauge invariant chiral fields are $x=\phi_{1}^{r+1} \phi_{2}^{r} \phi_{3}^{r-1} \ldots \phi_{r+2}^{0}, y=$ $\phi_{1}^{0} \phi_{2}^{1} \phi_{3}^{2} \ldots \phi_{r+2}^{r+1}$ and $z=\phi_{1} \phi_{2} \phi_{3} \ldots \phi_{r+2}$. They satisfy the $s u(r+1)$ singularity equation

$$
x y=z^{r+1}
$$

The deformation of this singularity can be given by the following D-terms

$$
\left|\phi_{a-1}\right|^{2}-2\left|\phi_{a}\right|^{2}+\left|\phi_{a+1}\right|^{2}=R^{a}
$$

\section{References}

[1] S. Katz, A. Klemm, C. Vafa, Geometric engineering of quantum field theories, Nucl. Phys. B497 (1997) 173, hep-th/9609239.

[2] S. Katz, P. Mayr, C. Vafa, Mirror symmetry and exact solution of $4 d N=2$ gauge theories I, Adv. Theor. Math. Phys. 1 (1998) 53, hep-th/9706110.

[3] C. Vafa, On N=1 Yang-Mills in Four Dimensions, Adv.Theor.Math.Phys. 2 (1998) 497503, hep-th/9801139.

[4] A. Belhaj, A. EL Fallah and E. H. Saidi, On the affine D4 mirror geometry; Class. Quantum. Grav. 16 (1999)3297-3306. On non simply laced mirror geometries in type II strings; Class. Quantum. Grav. 17 (1999)1-18.

[5] A. Belhaj, E. H. Saidi, Toric Geometry, Enhanced non Simply laced Gauge Symmetries in Superstrings and F-theory Compactifications, hep-th/0012131.

[6] A. Belhaj, L. B. Drissi, J. Rasmussen, On $N=1$ gauge models from geometric engineering in M-theory, Class.Quant.Grav. 20 (2003) 4973-4982, hep-th/0304019.

[7] T. J. Hollowood, A. Iqbal, C. Vafa, Matrix Models, Geometric Engineering and Elliptic Genera, hep-th/0310272.

[8] S. Katz, C. Vafa, Matter From Geometry, Nucl. Phys. B497(1997)146-154, hep-th/9606086.

[9] R. Ahl Laamara, M. Ait Ben Haddou, A. Belhaj, L. B. Drissi, E. H. Saidi, RG Cascades in Hyperbolic Quiver Gauge Theories, Nucl. Phys. B702(2004)163-188, hep-th/0405222. 
[10] M. Ait Ben Haddou, A. Belhaj, E. H. Saidi, Classification of N=2 supersymmetric CFT 4 : Indefinite Series, J.Phys. A38 (2005) 1793-1806, hep-th/0308005.

[11] M. Ait Ben Haddou, A. Belhaj, E. H. Saidi, Geometric Engineering of $N=2 C_{F} T_{4}$ based on Indefinite Singularities: Hyperbolic Case, Nucl. Phys. B674(2003)593-614, hep-th/0307244.

[12] J. J. Heckman, C. Vafa, From F-theory GUTs to the LHC, arXiv:0809.3452[hep-th].

[13] J. J. Heckman, C. Vafa, F-theory, GUTs, and the Weak Scale, arXiv:0809.1098[hep-th].

[14] J. J. Heckman, J. Marsano, N. Saulina, S. Schafer-Nameki, C. Vafa, Instantons and SUSY breaking in F-theory, arXiv:0808.1286[hep-th].

[15] J. Marsano, N. Saulina and S. Schafer-Nameki, Gauge Mediation in F-Theory GUT Models, arXiv:0808.1571 [hep-th].

[16] J. Marsano, N. Saulina and S. Schafer-Nameki, An Instanton Toolbox for F-Theory Model Building, arXiv:0808.2450 [hep-th].

[17] C. Beasley, J. J. Heckman, C. Vafa, GUTs and Exceptional Branes in F-theory - II: Experimental, arXiv:0806.0102[hep-th].

[18] M. Aganagic, C. Beem, Jihye Seo, C. Vafa, Extended Supersymmetric Moduli Space and a SUSY/Non-SUSY Duality, arXiv:0804.2489[hep-th].

[19] C. Beasley, J. J. Heckman, C. Vafa, GUTs and Exceptional Branes in F-theory - I, arXiv:0802.3391[hep-th].

[20] M. Wijnholt, F-Theory, GUTs and Chiral Matter, arXiv:0809.3878[hep-th].

[21] A. Font, L. E. Ibanez, Yukawa Structure from U(1) Fluxes in F-theory Grand Unification, arXiv:0811.2157[hep-th].

[22] J. L. Bourjaily, Local Models in F-Theory and M-Theory with Three Generations, arXiv:0901.3785[hep-th].

[23] B. S. Acharya, K. Bobkov, Kahler Independence of the G2-MSSM, arXiv:0810.3285[hepth]. 
[24] B. S. Acharya, K. Bobkov, G. L. Kane, P. Kumar, J. Shao, The $G_{2}-M S S M$ An $M$ Theory motivated model of Particle Physics, Phys. Rev. D78:065038,2008, arXiv:0801.0478[hep-th].

[25] B. Acharya, E. Witten, Chiral Fermions from Manifolds of $G_{2}$ Holonomy, arXiv:hep-th/0109152.

[26] A. Belhaj, E.H. Saidi, On HyperKahler Singularities, Mod. Phys. Lett. A 15(2000)1767, hep-th/0007143.

[27] C. Vafa, Evidence for F-theory, Nucl. Phys. B469 (1996) 403, hep-th/9602022.

[28] L. J. Boya, Arguments for F-theory, Mod.Phys.Lett. A21(2006)287-304, hep-th/0512047.

[29] E. Witten, Phases of $N=2$ theories in two dimensions, Nucl. Phys. B 403 (1993), 159, hep-th/9301042.

[30] R. Ahl Laamara, A. Belhaj, L.B. Drissi, E.H. Saidi, On Local Calabi-Yau Supermanifolds and Their Mirrors, J.Phys. A39(2006)5965-5978, hep-th/0601215.

[31] A. Belhaj, P. Diaz, A. Segui, On the Superstring Realization of the Yang Monopole, hep-th/0703255.

[32] A. Hanany, A. Iqbal, Quiver theories from D6-branes via mirror symmetry, JHEP 0204 (2002) 009, hep-th/010813.

[33] B. Feng, A. Hanany, Y.-H. He, A. Iqbal, Quiver theories, soliton spectra and PicardLefschetz transformations, hep-th/0206152.

[34] A.M. Uranga, Chiral four-dimensional string compactifications with intersecting D-branes, hep-th/0301032.

[35] V.G. Kac, Infinite Dimensional Lie Algebras, Third Edition, Cambridge University Press (1990).

[36] R. Ahl Laamara, A. Belhaj, L. J. Boya, L. Medari, A. Segui, On F-theory Quiver Models and Kac-Moody Algebras arXiv:0910.4852 [hep-th]

[37] R. Slansky, Group thoery for unified theory model building, Physics report, 79 No. 1 (1981)1-128. 